\title{
THE THEORY OF OPERATIONS ON BINARY RELATIONS
}

\author{
BY
}

T. TAMURA

1. Introduction. Let $G$ be a groupoid [2] and consider a congruence relation $\sigma$ on $G$ such that the factor groupoid $G / \sigma$ modulo $\sigma$ has a given type $\mathscr{T}$ where $\mathscr{T}$ is commutativity or idempotency or both. Then $\sigma$ is called a congruence of type $\mathscr{T}$ or $\mathscr{T}$-congruence. It is known that the set of all $\mathscr{T}$-congruences on $G$ has a smallest $\left({ }^{1}\right)$ element $\sigma_{0}$ with respect to the inclusion relation, that is, $\sigma_{0} \subseteq \sigma$ for all $\mathscr{T}$-congruences $\sigma$ on $G$. The smallest semilattice-congruence on semigroups was obtained by several people: by Clifford [15], McLean [16], by Kimura and the author [8], by Yamada [14] and recently, Petrich [6]. The general or abstract treatment was seen in the papers [4], [9] and [12]. After all, we can say that for any groupoid $G$ and for any type $\mathscr{T}$ expressed by implication condition or identity condition, the set of all $\mathscr{T}$-congruences on $G$ has a smallest element. Clifford and Preston interpreted these facts in [3] using the principle of the maximal homomorphic image.

The proof of the existence of the smallest congruence of type $\mathscr{T}$, or generally, of the smallest congruence of type $\mathscr{T}$ including a given relation $\rho$ is very easy, for we can see that the intersection of congruences of type $\mathscr{T}$ including $\rho$ is also a congruence of $\mathscr{T}$ including $\rho$. However, there is a problem asking how to construct the smallest congruence $\sigma_{0}$ by expanding $\rho$ instead of considering the intersection of all congruences of $\mathscr{T}$ including $\rho$. To do this, we introduce semiclosure operations on the system of relations in $\$ 2$. Especially if we provide the semi-closure operations with the condition "join-conservativeness," the procedure will be more convenient in the series of applications of the operations (§5). It is interesting that, with semi-closure operations, we can generalize the concept of systems of implications or identities to so large an extent that the concept of basic types can be introduced ( $\S 4)$. The argument in $\S 2$ and $\S 5$ will be effective on any complete lattice, not necessarily only the lattice of relations. The theory of operations on binary relations can be applied to many kinds of algebraic systems and probably other fields (§6). The operations on relations was already tried by the author in [12] and by Preston in a somewhat different

Presented to the Society August 29, 1963 under the title Operations on relations and their applications to groupoids; received by the editors January 17, 1964 and, in revised form, May 3, 1965.

(1) An element $a$ of a partially ordered set $E$ is called "smallest" if $a \leqq x$ for all $x \in E$. Then a smallest element $a$ is unique. A "smallest" element is equivalent to a "unique minimal" element. (See p. 7 in [1].) 
problem [7], but this paper simplifies, resystematizes and also generalizes the theory in [12].

A shorter version of this paper has already been published, without proof, in [13]. A few notations used in [13] are modified in this paper.

2. Semi-closure operations. Let $E$ be a nonempty set. A binary relation $\rho$ on $E$ is defined to be a subset of the product set $E \times E$. Let $\mathscr{B}$ be a complete lattice composed of binary relations with respect to the usual inclusion relation $\subseteq$. Let $\mathscr{B}_{0}$ denote the complete lattice of all binary relations, together with the empty relation, on $E . \mathscr{B}$ is a subset of $\mathscr{B}_{0}$ and a lattice with the same inclusion as in $\mathscr{B}_{0}$. For an arbitrary subset $\mathscr{A}=\left\{\rho_{\alpha} ; \alpha \in \Gamma\right\}$ of $\mathscr{B}$, the join (or union) and meet (or intersection) are denoted by

$$
\begin{aligned}
& \bigcup_{\alpha \in \Gamma} \rho_{\alpha} \text { or } \bigcup_{\alpha} \rho_{\alpha} \text { or } \mathfrak{I}(\mathscr{A}) \text { or } \bigcup\left\{\rho_{\alpha} ; \alpha \in \Gamma\right\}, \\
& \bigcap_{\alpha \in \Gamma} \rho_{\alpha} \text { or } \bigcap_{\alpha} \rho_{\alpha} \text { or } \mathfrak{M}(\mathscr{A}) \text { or } \bigcap\left\{\rho_{\alpha} ; \alpha \in \Gamma\right\},
\end{aligned}
$$

respectively. Consider an unary operation $P$, that is, a mapping of $\mathscr{B}$ into itself: $\rho \rightarrow \rho P$. The inclusion relation with respect to the operations is defined as follows:

$$
P \leqq Q \text { if and only if } \rho P \subseteq \rho Q \text { for all } \rho \in \mathscr{B} .
$$

Hence $P=Q$ if and only if $P \leqq Q$ and $Q \leqq P$, i.e., $\rho P=\rho Q$ for all $\rho \in \mathscr{B}$. Accordingly, the join and meet of a set $\left\{P_{\xi} ; \xi \in \Xi\right\}$ are defined as follows:

$$
\rho\left(\bigcup_{\xi} P_{\xi}\right)=\bigcup_{\xi} \rho P_{\xi}, \quad \rho\left(\bigcap_{\xi} P_{\xi}\right)=\bigcap_{\xi} \rho P_{\xi} .
$$

We consider the empty operation $\mathbf{a}$, that is, the operation which maps all relations belonging to $\mathscr{B}$ to the empty relation $\square: \rho \square=\square$ for all $\rho \in \mathscr{B}$.

A multiplication of operations $P$ and $Q$ is defined by

$$
P Q: \rho(P Q)=(\rho P) Q \text {. }
$$

Following Birkoff and Ore [1], [5], we give the following definitions:

(2.4.1) $P$ is called isotonic if $\rho \subseteq \sigma$ implies $\rho P \subseteq \sigma P$.

(2.4.2) $P$ is called extensive if $\rho \subseteq \rho P$ for all $\rho \in \mathscr{B}$.

(2.4.3) $P$ is called idempotent if $P^{2}=P$.

Definition. An isotonic, extensive operation is called a semi-closure operation, and an idempotent semi-closure operation is called a closure operation.

The set of all semi-closure operations on $\mathscr{B}$ is denoted by $\mathfrak{P}$. Immediately from the definition we have:

LEMMA 2.1. $\mathfrak{B}$ is a complete lattice with respect to (2.1) or (2.2), and at the same time is a partially ordered semigroup with respect to (2.3) and (2.1). 
Proof. We can prove the following: If each $P_{\xi}$ is isotonic, then both $\bigcup_{\xi} P_{\xi}$ and $\bigcap_{\xi} P_{\xi}$ are isotonic; if $P_{\xi}$ is extensive, then both $\bigcup_{\xi} P_{\xi}$ and $\bigcap_{\xi} P_{\xi}$ are extensive; if $P$ and $Q$ are isotonic, then $P Q$ is isotonic; if $P$ and $Q$ are extensive, then $P Q$ is extensive. Clearly, $P \leqq Q$ implies $U P \leqq U Q$ for all $U \in \mathfrak{P}$; and if $P \leqq Q$, then $P U \leqq Q U$ by isotonicity. The greatest element of $\mathfrak{P}$ is $P_{0}, \rho P_{0}=\omega,(\omega$ is the greatest element of $\mathscr{B})$, for all $\rho \in \mathscr{B}$; the smallest element of $\mathfrak{P}$ is $P_{1}, \rho P_{1}=\rho$ for all $\rho \in \mathscr{B}$.

A relation $\rho$ is called $P$-closed if $\rho P=\rho$.

LEMMA 2.2. Let $\left\{P_{\xi} ; \xi \in \Xi\right\}$ be a set of semi-closure operations on $\mathscr{B}$. A relation $\rho$ is $P_{\xi}$-closed for all $\xi \in \Xi$ if and only if $\rho$ is $\left(\bigcup_{\xi \in \Xi} P_{\xi}\right)$-closed.

Proof. If $\rho$ is $P_{\xi}$-closed for all $\xi \in \Xi$, by the definition,

$$
\rho\left(\bigcup_{\xi} P_{\xi}\right)=\bigcup_{\xi} \rho P_{\xi}=\rho .
$$

Conversely, if $\rho$ is $\left(\bigcup_{\xi} P_{\xi}\right)$-closed,

$$
\rho \subseteq \rho P_{\xi} \subseteq \rho\left(\bigcup_{\xi} P_{\xi}\right)=\rho \text { or } \rho P_{\xi}=\rho \text { for all } \xi \in \Xi .
$$

Semi-closure operations can be defined in any complete lattice in the same way, and all the discussions so far are effective to any case. Since $\mathfrak{P}$ is a complete lattice, the mapping $P \rightarrow P Q$, for a fixed $Q \in \mathfrak{P}$, is a semi-closure operation on $\mathfrak{P}$. If $P Q=P, P$ is called $Q$-closed.

Easily we can prove

Lemma 2.3. If $P$ is $Q$-closed, then $P \geqq Q$. If $P \geqq Q$ and $P$ is a closure operation, then $P$ is $Q$-closed.

Proof. For the first part of the lemma, $P=P Q \geqq Q$; for the second, $P=P^{2} \geqq P Q \geqq P$; q.e.d.

For a subset $\left\{P_{\xi} ; \xi \in \Xi\right\}$ of $\mathfrak{P}$ and for any relation $\rho \in \mathscr{B}$, define

$$
\mathscr{B}_{\rho}=\left\{\sigma \in \mathscr{B} ; \sigma \supseteq \rho, \sigma P_{\xi}=\sigma \text { for all } \xi \in \Xi\right\} \text {. }
$$

LEMMA 2.4. The following three statements are equivalent:

(2.6.1) $\rho H=\mathfrak{M}\left(\mathscr{B}_{\rho}\right)$.

(2.6.2) $H$ is the smallest $P_{\xi}$-closed semi-closure operation for all $\xi \in \Xi$.

(2.6.3) $H$ is the smallest closure operation such that $H \geqq P_{\xi}$ for all $\xi \in \Xi$.

Proof. We may show $(2.6 .1) \Rightarrow(2.6 .2)$, and $(2.6 .1) \Rightarrow(2.6 .3)$. Because of the uniqueness of the smallest closure operation, the converses are obvious. Suppose $H$ is defined by (2.6.1). We shall prove (2.6.1) $\Rightarrow(2.6 .2) . H$ is isotonic: if $\rho_{1} \subseteq \rho_{2}$, then $\mathscr{B}_{\rho_{2}} \subseteq \mathscr{B}_{\rho_{1}}$, and so $\rho_{1} H \subseteq \rho_{2} H$. Extensiveness is clear. We prove that $H$ is $P_{\xi}$-closed. Since $\rho H \subseteq \sigma$ for all $\sigma \in \mathscr{B}_{\rho}$, 


$$
\rho H P_{\xi} \subseteq \sigma P_{\xi}=\sigma \text { for all } \sigma \in \mathscr{B}_{\rho}
$$

and so $\rho H P_{\xi} \subseteq \rho H$, while $\rho H \subseteq \rho H P_{\xi}$, and hence $H P_{\xi}=H$. To show that $H$ is the smallest, let $K$ be any semi-closure operation such that $K P_{\xi}=K$ for all $\xi$. This means

$$
\rho K \in \mathscr{B}_{\rho} \text { and hence } \rho H \subseteq \rho K \text { or } H \leqq K \text {. }
$$

Thus (2.6.2) has been proved. For the proof of (2.6.3) we show $H^{2}=H$. Since $H P_{\xi}=H$, we see

$$
\rho H \in \mathscr{B}_{\rho H}=\left\{\sigma ; \sigma \supseteq \rho H, \sigma P_{\xi}=\sigma\right\}
$$

whence $(\rho H) H \subseteq \rho H$, i.e., $H^{2} \leqq H$. It is obvious that $H$ is the smallest closure operation including $P_{\xi}$ for all $\xi \in \Xi$, q.e.d.

$H$ is called the closure operation generated by $\left\{P_{\xi} ; \xi \in \Xi\right\}$ and denoted by $H=\left\{P_{\xi} ; \xi \in \Xi\right\}^{\#}$; if $\left\{P_{\xi} ; \xi \in \Xi\right\}$ consists of $P$ alone, it is denoted by $H=P^{\#}$. Lemma 2.4 shows

$$
\begin{aligned}
\left\{P_{\xi} ; \xi \in \Xi\right\}^{\#} & =\bigcap\left\{Q \in \mathfrak{P} ; Q P_{\xi}=Q \text { for all } \xi \in \Xi\right\} \\
& =\bigcap\left\{Q \in \mathfrak{P} ; Q^{2}=Q, Q \geqq P_{\xi} \text { for all } \xi \in \Xi\right\} .
\end{aligned}
$$

LEMMA 2.5. The mapping $P \rightarrow P^{\#}$ is a closure operation on $\mathfrak{P}$, that is,

$$
\left(P^{\#}\right)^{\#}=P^{\#} \text {. }
$$

Proof. Since $P^{\#}$ is clearly the smallest closure operation containing $P^{\#}$. It is immediate from (2.6.3) that $\left(P^{\#}\right)^{\#}=P^{\#}$.

THEOREM 2.1. $H=\left\{P_{\xi} ; \xi \in \Xi\right\}^{\#}=\left(\bigcup_{\xi} P_{\xi}\right)^{\#}=\left(\bigcup_{\xi} P_{\xi}^{\#}\right)^{\#}$

Proof. By Lemmas 2.2, 2.4 or (2.7), we have $\left\{P_{\xi} ; \xi \in \Xi\right\}^{\#}=\left(\bigcup_{\xi} P_{\xi}\right)^{\#}$. Since $P_{\xi}^{\#} \leqq\left(\bigcup_{\xi} P_{\xi}\right)^{\#}$ for all $\xi \in \Xi, \bigcup_{\xi} P_{\xi}^{\#} \leqq\left(\bigcup_{\xi} P_{\xi}\right)^{\#}$ and hence

$$
\left(\bigcup_{\xi} P_{\xi}^{\#}\right)^{\#} \leqq\left(\bigcup_{\xi} P_{\xi}\right)^{\# \#}=\left(\bigcup_{\xi} P_{\xi}\right)^{\#}
$$

by Lemma 2.5 . The inequality in the other direction is easily obtained. q.e.d.

Thus if $P$ is a semi-closure operation, $\rho P^{\#}$ is the smallest $P$-closed relation including $\rho$.

Let

$$
\mathscr{B} P^{\#}=\left\{\rho P^{\#} ; \rho \in \mathscr{B}\right\}
$$

Then

$$
\mathscr{B} P^{\#}=\left\{\rho \in \mathscr{B} ; \rho P^{\#}=\rho\right\}=\{\rho \in \mathscr{B} ; \rho P=\rho\} .
$$

REMARK. So far we have dealt with the semi-closure operations on any $\mathscr{B}$, but the treatment of the operations on the set $\mathscr{B}_{0}$ of all binary relations is of 
important significance. Such a special treatment does not lose generality; the reason will be clarified in the next section.

3. Operations on subsets. Let $\mathscr{B}_{1}$ and $\mathscr{B}_{2}$ be two complete lattices in which the meet ordering in $\mathscr{B}_{i}$ are respectively symbolized by

$$
\bigcap_{i}, \subseteq_{i}(i=1,2) .
$$

$\mathscr{B}_{1}$ is called order-invariant in $\mathscr{B}_{2}$ if the following two conditions are satisfied $\mathscr{B}_{1}$ is a subset of $\mathscr{B}_{2}$.

$\mathscr{B}_{1}$ is called meet-invariant in $\mathscr{B}_{2}$ if (3.2) of the above two is replaced by (3.3) below:

$$
\text { If } \rho_{\xi} \in \mathscr{B}_{1}, \xi \in \Xi \text {, then } \bigcap_{\xi} \rho_{\xi}=\bigcap_{\xi} \rho_{\xi} \text {. }
$$

Obviously (3.3) implies (3.2). If $\mathscr{B}_{1}$ is order-invariant or meet-invariant in $\mathscr{B}_{2}$, we will use the symbol $\subseteq$ or $\bigcap$ in $\mathscr{B}_{i}$ instead of $\subseteq_{i}$ or $\bigcap_{i}$ respectively, $(i=1,2)$.

Let $\mathfrak{P}_{i}$ be the complete lattice of all semi-closure operations on $\mathscr{B}_{i}(i=1,2)$, and $\mathfrak{P}_{\mathscr{B}_{1}}$ the set of all semi-closure operations $Q$ on $\mathscr{B}_{2}$ such that $\mathscr{B}_{1} Q \subseteq_{1} \mathscr{B}_{1}$. $\mathfrak{P}_{\mathscr{B}_{1}}$ is a partially ordered semigroup (Lemma 2.1).

THEOREM 3.1. Suppose that $\mathscr{B}_{1}$ is order-invariant in $\mathscr{B}_{2}$. Then the restriction of any $Q$ in $\mathfrak{P}_{\mathscr{B}_{1}}$ to $\mathscr{B}_{1}$ is a semi-closure operation on $\mathscr{B}_{1}$, and any semiclosure operation on $\mathscr{B}_{1}$ is obtained in this manner. $\mathfrak{P}_{\mathscr{B}_{1}}$ contains a lattice $\mathfrak{P}_{1}^{\prime}$ (not necessarily a sublattice of $\mathfrak{P}_{2}$ ) which is the homomorphic image of $\mathfrak{P}_{\mathscr{B}_{1}}$ as partially ordered semigroups and which is isomorphic onto $\mathfrak{P}_{1}$ as lattices and as partially ordered semigroups.

Proof. Let $Q \in \mathfrak{P}_{\mathscr{B}_{1}}$. The restriction of $Q$ to $\mathscr{B}_{1}$ is denoted by $Q_{1}$, namely $\sigma Q_{1}=\sigma Q$ for all $\sigma \in \mathscr{B}_{1}$.

(3.2) assures that $Q_{1} \in \mathfrak{P}_{1}$; also the conditions (3.2) and $\mathscr{B}_{1} Q \subseteq \mathscr{B}_{1}$ enable us to prove:

$$
Q \leqq R \text { in } \mathfrak{P}_{\mathscr{B}_{1}} \text { implies } Q_{1} \leqq R_{1} \text { in } \mathfrak{P}_{1}
$$

$$
(Q R)_{1}=Q_{1} R_{1} \text {. }
$$

Let $P \in \mathfrak{P}_{1}$. For $P$, we define $P^{*}$ on $\mathscr{B}_{2}$ as follows:

$$
\sigma P^{*}= \begin{cases}\bigcap_{1}\left\{\rho P ; \sigma \subseteq \rho \in \mathscr{B}_{1}\right\} & \text { if }\left\{\rho \in \mathscr{B}_{1} ; \sigma \subseteq \rho\right\} \neq \square, \\ \omega_{2} & \text { otherwise, }\end{cases}
$$


where $\omega_{2}$ is the greatest element of $\mathscr{B}_{2}$. It can be shown without difficulty that $P^{*} \in \mathfrak{P}_{2}$. If $\sigma \in \mathscr{B}_{1}$, then $\sigma P \subseteq \rho P$ for all $\rho$ such that $\sigma \subseteq \rho \in \mathscr{B}_{1}$; this means that $\sigma \in \mathscr{B}_{1}$ implies $\sigma P^{*}=\sigma P, \mathscr{B}_{1} P^{*} \subseteq \mathscr{B}_{1}$; that is, $P^{*} \in \mathfrak{P}_{\mathscr{B}_{1}}$ and

$$
\left(P^{*}\right)_{1}=P \text {. }
$$

Thus we have proved that the mapping $Q \rightarrow Q_{1}$ is the homomorphism of $\mathfrak{P}_{\mathscr{B}_{1}}$ onto $\mathfrak{P}_{1}$ as partially ordered semigroups.

Let $\mathfrak{P}_{1}^{\prime}=\left\{P^{*} ; P \in \mathfrak{P}_{1}\right\}$. If $P, R$ in $\mathfrak{P}_{1}$ and $P \leqq R$, the definition (3.6) and (3.2) immediately give $\sigma P^{*} \subseteq \sigma R^{*}$ for all $\sigma \in \mathscr{B}_{2}$, or $P^{*} \leqq R^{*}$ in $\mathfrak{P}_{1}^{\prime}$. Conversely if $P^{*} \leqq R^{*}$ in $\mathfrak{P}_{1}^{\prime}$, then, particularly for $\sigma \in \mathscr{B}_{1}, \sigma P^{*} \subseteq \sigma R^{*}$, namely $P \leqq R$ in $\mathfrak{P}_{1}$; hence

$$
P \leqq R \text { in } \mathfrak{P}_{1} \text { if and only if } P^{*} \leqq R^{*} \text { in } \mathfrak{P}_{1}^{\prime} \text {. }
$$

Furthermore, by (3.7) and the homomorphism $Q \rightarrow Q_{1}$, we have

$$
\begin{aligned}
\left(P^{*} Q^{*}\right)_{1} & =\left(P^{*}\right)_{1}\left(Q^{*}\right)_{1}=P Q \\
P^{*} Q^{*} & =(P Q)^{*} .
\end{aligned}
$$

Thus (3.7), (3.8) and (3.9) show that the mapping $P \rightarrow P^{*}$ is the isomorphism of $\mathfrak{P}_{1}$ onto $\mathfrak{P}_{1}^{\prime}$ not only as partially ordered semigroups but also as lattices. We have the series of homomorphisms: $\mathfrak{P}_{\mathscr{R}_{1}} \rightarrow \mathfrak{P}_{1} \rightarrow \mathfrak{P}_{1}^{\prime}$, accordingly $\mathfrak{P}_{\mathscr{B}_{1}}$ is homomorphic onto $\mathfrak{P}_{1}^{\prime}$. The homomorphism $\mathfrak{P}_{\mathscr{B}_{1}} \rightarrow \mathfrak{P}_{1}^{\prime}$ is explicitly expressed by the mapping $Q \rightarrow Q^{\prime}$ as follows: For $Q \in \mathfrak{P}_{\mathscr{B}_{1}}$

$$
\sigma Q^{\prime}= \begin{cases}\bigcap_{1}\left\{\rho Q ; \sigma \subseteq \rho \in \mathscr{B}_{1}\right\} & \text { if }\left\{\rho \in \mathscr{B}_{1} ; \sigma \subseteq \rho\right\} \neq \square, \\ \omega_{2} & \text { otherwise. }\end{cases}
$$

The proof of the theorem is finished.

Additionally, we easily have

COROLlary. If $\mathscr{B}_{1}$ is meet-invariant in $\mathscr{B}_{2}$, then $\mathfrak{P}_{1}^{\prime}$ is meet-invariant in $\mathfrak{P}_{2}$.

4. Types of relations. Recall that $\mathscr{B}_{0}$ denotes the complete lattice of all binary relations on a set $E$.

A subset $\mathscr{T}$ of $\mathscr{B}_{0}$ is called a type of relations on $E$ if and only if

$$
\omega \in \mathscr{T} \text {, where } \omega \text { is the greatest element of } \mathscr{B}_{0} .
$$

For convenience we shall call it "a type of relations on $E$ " or "a type on $E$ " and if $E$ is fixed, we may call it simply "a type." An element of $\mathscr{T}$ is called a "relation of type $\mathscr{T}$."

A type on $E$ is called a basic type on $E$ if and only if

$$
\mathscr{T} \text { is a complete lattice in } \mathscr{B}_{0}-\{\square\} .
$$


In other words, a basic type on $E$ is a subset of $\mathscr{B}_{0}$ satisfying (4.1) and (4.2). In these conditions, (4.2) can be replaced by:

$$
\text { For any subset }\left\{\rho_{\alpha} ; \alpha \in A\right\} \text { of } \mathscr{T}, \square \neq \bigcap_{\alpha \in A} \rho_{\alpha} \in \mathscr{T} \text {. }
$$

Also (4.1) can be replaced by:

$$
\text { For any } \rho \in \mathscr{B}_{0} \text { there is a } \sigma \in \mathscr{T} \text { such that } \rho \subseteq \sigma .
$$

After all $\{(4.1)$ and $(4.2)\} \rightleftarrows\left\{(4.1)\right.$ and $\left.\left(4.2^{\prime}\right)\right\} \rightleftarrows\left\{\left(4.1^{\prime}\right)\right.$ and $\left.\left(4.2^{\prime}\right)\right\}$.

Clearly the set of all types on $E$ is a complete sublattice of the power set of $\mathscr{B}_{0}$, but the set of all basic types is not. The following theorem is easily obtained.

THEOREM 4.1. The intersection of arbitrary number of basic types is also a basic type. Any type is a set-union of basic types.

The following theorem closely connects the concept of basic types with that of closure operations; it is an application of the known result in [1], [5] to the concept of basic types. For the reader's convenience, we give a brief proof.

THEOREM 4.2. A type $\mathscr{T}$ on a set $E$ is a basic type if and only if there is a semi-closure operation $P$ on $\mathscr{B}_{0}$ such that $\mathscr{T}=\mathscr{B}_{0} P^{\#}$. There is a one-to-one correspondence between all the closure operations $P$ on $\mathscr{B}_{0}$ and all the basic types $\mathscr{T}_{P}$ on $E$ under the mapping $P \rightarrow \mathscr{T}_{P}, \mathscr{T}_{P}=\mathscr{B}_{0} P$, such that, for any number of closure operations $P_{\xi}$ on $\mathscr{B}_{0}$,

$$
\mathscr{B}_{0}\left(\bigcap_{\xi} P_{\xi}\right)=\bigcap_{\xi} \mathscr{T}_{P_{\xi}} \cdot
$$

Proof. For a semi-closure operation $P$ on $\mathscr{B}_{0}$, a subset $\mathscr{T}_{P}$ of $\mathscr{B}_{0}$ is defined to be

$$
\mathscr{T}_{P}=\left\{\rho \in \mathscr{B}_{0} ; \rho P=\rho\right\} .
$$

Then $\omega \in \mathscr{T}_{\boldsymbol{P}}$, and if $\rho_{\alpha} \in \mathscr{T}_{\boldsymbol{P}}$, then $\left(\bigcap_{\alpha} \rho_{\alpha}\right) P=\bigcap_{\alpha} \rho_{\alpha} P$; therefore $\mathscr{T}_{\boldsymbol{P}}$ is a basic type on $E$. By (2.9), $\mathscr{T}_{P}=\mathscr{T}_{P \#}$, from which we immediately have $\mathscr{T}_{P *}=\mathscr{B}_{0} P^{\#}$. Also we see that if $P^{\#}=Q^{\#}$ then $\mathscr{T}_{P}=\mathscr{T}_{Q}$.

Conversely, let $\mathscr{T}$ be a basic type on $E$. An operation $P$ on $\mathscr{B}_{0}$ is defined as follows:

$$
\rho P=\bigcap\{\sigma \in \mathscr{T} ; \rho \subseteq \sigma\} .
$$

Then it can be shown that $P$ is a closure operation on $\mathscr{B}_{0}$ and $\mathscr{T}=\mathscr{B}_{0} P$. (4.3) is immediately obtained from the definition of the meet of operations. q. e.d.

The smallest relation of type $\mathscr{T}$ containing $\rho \in \mathscr{B}_{0}$, if it exists, is called the relation of type $\mathscr{T}$ generated by $\rho$. It always exists if $\mathscr{T}$ is a basic type.

Let $\left\{P_{\xi} ; \xi \in \Xi\right\}$ be a family of semi-closure operations on $\mathscr{B}_{0}$. A relation $\rho$ will be said to be of type $\bigwedge_{\xi} P_{\xi}$ if it is $P_{\xi}$-closed for every $\xi$ in $\Xi$, and of type 
$\bigvee_{\xi} P_{\xi}$ if it is $P_{\xi}$-closed for some $\xi$ in $\Xi$. The set of all relations $\rho$ of type $\bigwedge_{\xi} P_{\xi}$ is a basic type because of Lemmas 2.1 and 2.2. On the other hand, the set of all relations of type $\bigvee_{\xi} P_{\xi}$ is not necessarily a basic type; to distinguish it we call it a join-type.

THEOREM 4.3. Let $\rho$ be any relation belonging to $\mathscr{B}_{0}$ and let $\left\{P_{\xi} ; \xi \in \Xi\right\}$ be a system of semi-closure operations on $\mathscr{B}_{0}$. There exists a unique relation $\sigma$ of type $\bigwedge_{\xi} P_{\xi}$ generated by $\rho$, and $\sigma=\rho H, H$ given in Lemma 2.4.

Proof. This is an immediate consequence of Lemmas 2.2 and 2.4, or Theorem 2.1.

THEOREM 4.4. If $|\Xi|>1$, then there exists a relation $\sigma$ of type $\bigvee_{\xi} P_{\xi}$ generated by a given relation $\rho$ if and only if $\rho P^{\prime}$ is of type $\bigvee_{\xi} P_{\xi}$ where $P^{\prime}=\bigcap_{\xi} P_{\xi}^{\#}$. If it exists, $\sigma=\rho P^{\prime}$.

Proof. Suppose $\sigma$ is of join-type $\bigvee_{\xi} P_{\xi},|\Xi|>1$, generated by $\rho$. Then $\sigma$ is $P_{\xi_{0}}$-closed for some $\xi_{0} \in \Xi$. Then, since each $\rho P_{\xi}^{\#}$ is of type $\bigvee_{\xi} P_{\xi}$,

$$
\rho P_{\xi_{0}}^{\#} \subseteq \sigma \subseteq \rho P_{\xi}^{\#} \text { for all } \xi \in \Xi,
$$

whence we have $\sigma=\rho P_{\xi_{0}}^{\#}=\rho \bigcap_{\xi} P_{\xi}^{\#}$.

Conversely, suppose $\sigma=\rho\left(\bigcap_{\xi} P_{\xi}^{\xi}\right)$ is of type $\bigvee_{\xi} P_{\xi}$. Let $\eta$ be any relation of type $\bigvee_{\xi} P_{\xi}$ containing $\rho$, that is, $\eta P_{\xi_{1}}^{\#}=\eta$ for some $\xi_{1} \in \Xi$. Then we get

$$
\eta=\eta P_{\xi_{1}}^{\#} \supseteq \rho P_{\xi_{1}}^{\#} \supseteq \rho \bigcap_{\xi} P_{\xi}^{\#}=\sigma .
$$

Thus we have proved that $\sigma$ is the relation of type $\bigvee_{\xi} P_{\xi}$ generated by $\rho$.

So far we have considered types of relations on $E$ for fixed $E$. Now we can generalize this concept to the case of collections of $E_{\lambda}$. q. e.d.

Let $\subseteq$ be a collection of sets $E_{\lambda}, \lambda \in \Lambda: \subseteq=\left\{E_{\lambda} ; \lambda \in \Lambda\right\}$.

A type $\mathscr{T}$ of relations on $\mathcal{S}$ is defined to be a collection of types $\mathscr{T}_{\lambda}$ of relations on $E_{\lambda}$ :

$$
\mathscr{T}=\left\{\mathscr{T}_{\lambda} ; \lambda \in \Lambda\right\} .
$$

For a set of types $\mathscr{T}^{(\xi)}$ on $\mathfrak{S}$, the meet and join are defined as follows:

$$
\begin{gathered}
\text { if } \mathscr{T}^{(\xi)}=\left\{\mathscr{T}_{\lambda}^{(\xi)} ; \lambda \in \Lambda\right\}, \xi \in \Xi \\
\bigcap_{\xi \in \Xi} \mathscr{T}^{(\xi)}=\left\{\bigcap_{\xi \in \Xi} \mathscr{T}_{\lambda}^{(\xi)} ; \lambda \in \Lambda\right\}, \bigcup_{\xi \in \Xi} \mathscr{T}^{(\xi)}=\left\{\bigcup_{\xi \in \Xi} \mathscr{T}_{\lambda}^{(\xi)} ; \lambda \in \Lambda\right\} .
\end{gathered}
$$

The set of all types on $\subseteq$ is a complete lattice; a basic type on $\subseteq$ is also defined to be the collection of basic types on $E_{\lambda}$. All the statements with respect to the types on $E$ can be redescribed as those with respect to $\mathfrak{S}_{\text {. }}$

The types induced by implications or identities are regarded as the types on $\subseteq$. For example, if $\mathfrak{S}$ is the collection of all semigroups, then the concept of types 
of congruence relations on $\mathfrak{I}$ is connected with the concept of the so-called "type of semigroups," something like commutative semigroups, cancellative semigroups, etc.

5. Join-conservativeness. In this section we return to the discussion on the basic types $\bigwedge_{\xi} P_{\xi}$. If the additional condition, join-conservativeness, is provided, the closure operation $\left\{P_{\xi} ; \xi \in \Xi\right\}^{\#}$ can be given in a more convenient, simple, explicit form.

A subset $\mathscr{A}$ of a complete lattice $\mathscr{B}$ is called a directed subset of $\mathscr{B}$ if, for any $\rho, \sigma \in \mathscr{A}$, there is a $\tau \in \mathscr{A}$ such that $\rho \cup \sigma \subseteq \tau$. An operation $P$ is called joinconservative if, for any directed subset $\mathscr{A}$ of $\mathscr{B}$,

$$
[\mathfrak{I}(\mathscr{A})] P \subseteq \mathfrak{I}(\mathscr{A} P) \text { where } \mathscr{A} P=\{\rho P ; \rho \in \mathscr{A}\} .
$$

LEMMA 5.1. If each $P_{\xi}$ is join-conservative, $\bigcup_{\xi \in \Xi} P_{\xi}$ is join-conservative.

Proof. Let $\left\{\rho_{\alpha} ; \alpha \in \Lambda\right\}$ be a directed subset of $\mathscr{B}$.

$$
\left[\bigcup_{\alpha} \rho_{\alpha}\right]\left(\bigcup_{\xi} P_{\xi}\right)=\bigcup_{\xi}\left[\bigcup_{\alpha} \rho_{\alpha}\right] P_{\xi} \subseteq \bigcup_{\xi}\left[\bigcup_{\alpha} \rho_{\alpha} P_{\xi}\right]=\bigcup_{\alpha}\left[\bigcup_{\xi} \rho_{\alpha} P_{\xi}\right]=\bigcup_{\alpha}\left[\rho_{\alpha} \bigcup_{\xi} P_{\xi}\right] .
$$

Lemma 5.2. If $P$ and $Q$ are isotonic and join-conservative, then $P Q$ is joinconservative.

Proof. Let $\left\{\rho_{\alpha} ; \alpha \in \Lambda\right\}$ be a directed subset. Then $\left\{\rho_{\alpha} P ; \alpha \in \Lambda\right\}$ is also directed, since if $\rho_{\alpha} \cup \rho_{\beta} \subseteq \rho_{\gamma}$ for some $\gamma, \rho_{\alpha} P \cup \rho_{\beta} P \subseteq\left(\rho_{\alpha} \cup \rho_{\beta}\right) P \subseteq \rho_{\gamma} P$. Therefore,

$$
\left[\bigcup_{\alpha} \rho_{\alpha}\right] P Q=\left\{\left[\bigcup_{\alpha} \rho_{\alpha}\right] P\right\} Q \subseteq\left[\bigcup_{\alpha}\left(\rho_{\alpha} P\right)\right] Q \subseteq \bigcup_{\alpha}\left(\rho_{\alpha} P\right) Q .
$$

Thus the set of all isotonic join-conservative operations on $\mathscr{B}$ is a semigroup with respect to the multiplication (2.3). If $P$ is isotonic and join-conservative, then (5.1) is equivalent to

$$
[\mathfrak{I}(\mathscr{A})] P=\mathfrak{I}(\mathscr{A} P) .
$$

We remark that we can define join-conservative operations on $\mathfrak{P}$ in the same way; and if $P$ is a join-conservative operation on $\mathscr{B}$, then it is also a join-conservative operation on $\mathfrak{P}$ in the sense that $Q \rightarrow Q P, Q \in \mathfrak{P}$.

THEOREM 5.1. Let $\left\{P_{\xi} ; \xi \in \Xi\right\}$ be a set of join-conservative semi-closure operations. Then the closure operation generated by this set is expressed in the union of all the products of finite number of $P_{\xi}$ 's:

$$
\left\{P_{\xi} ; \xi \in \Xi\right\}^{\#}=\bigcup_{\xi, n, k} P_{\xi_{1}}^{n_{1}} \cdots P_{\xi_{k}}^{n_{k}}
$$

where the products run through all $\left(\xi_{1}, \cdots, \xi_{k}\right)$, all $\left(n_{1}, \cdots, n_{k}\right), \xi_{i} \in \Xi, n_{i}$ positive integers, $k=1,2, \cdots$. 
Proof. Let $P=\bigcup_{\xi, n, k} P_{\xi_{1}}^{n_{1}} \cdots P_{\xi_{k}}^{n_{k}}$. By Lemmas 5.2 and 5.1, $P$ is a join-conservative semi-closure operation. First we shall prove that $P$ is $P_{\xi}$-closed. Since the set of all the forms $P_{\xi_{1}}^{n_{1}} \ldots P_{\xi_{k}}^{n_{k}}$ is a directed subset of $\mathfrak{P}$, we get

$$
P \leqq P P_{\xi}=\left[\bigcup_{\xi, n, k} P_{\xi_{1}}^{n_{1}} \cdots P_{\xi_{k}}^{n_{k}}\right] P_{\xi}=\bigcup_{\xi, n, k} P_{\xi_{1}}^{n_{1}} \cdots P_{\xi_{k}}^{n_{k}} P_{\xi} \leqq P .
$$

Therefore, $P P_{\xi}=P$ for all $\xi \in \Xi$. Next, to show that $P$ is smallest, let $P^{\prime}$ be any $P_{\xi}$-closed semi-closure operation for all $\xi \in \Xi: P^{\prime} P_{\xi}=P^{\prime}$. Then

and so

$$
P_{\xi_{1}}^{n_{1}} \cdots P_{\xi_{k}}^{n_{k}} \leqq P^{\prime} P_{\xi_{1}}^{n_{1}} \cdots P_{\xi_{k}}^{n_{k}}=P^{\prime}
$$

$$
P=\bigcup_{\xi, n, k} P_{\xi_{1}}^{n_{1}} \cdots P_{\xi_{k}}^{n_{k}} \leqq P^{\prime}
$$

By Lemma 2.4, we have proved $P=\left\{P_{\xi} ; \xi \in \Xi\right\}^{\#}$.

THEOREM 5.2. If $\Xi$ is finite, namely, if $P_{1}, \cdots, P_{n}$ are join-conservative semiclosure operations, then for every permutation $\left(i_{1}, \cdots, i_{n}\right)$ of the letters $1, \cdots, n$,

$$
\left\{P_{i} ; i=1,2, \cdots, n\right\}^{\#}=\bigcup_{m=1}^{\infty}\left(P_{i_{1}} \cdots P_{i_{n}}\right)^{m} .
$$

Proof. We use the result of Theorem 5.1. Let $P_{\xi_{j}}$ be one of $P_{1}, \cdots, P_{n}$. Since $P_{\xi_{1}}^{n_{1}} \cdots P_{\xi_{k}}^{n_{k}} \leqq\left(P_{i_{1}} \cdots P_{i_{n}}\right)^{n_{1}} \cdots\left(P_{i_{1}} \cdots P_{i_{n}}\right)^{n_{k}}=\left(P_{i_{1}} \cdots P_{i_{n}}\right)^{n_{1}+\ldots+n_{k}}$, we have

$$
\left\{P_{i} ; i=1, \cdots, n\right\}^{\#}=\bigcup_{\xi, n, k} P_{\xi_{1}}^{n_{1}} \cdots P_{\xi_{k}}^{n_{k}} \leqq \bigcup_{m=1}^{\infty}\left(P_{i_{1}} \cdots P_{i_{n}}\right)^{m} \leqq\left\{P_{i} ; i=1, \cdots, n\right\}^{\#}
$$

whence we get (5.4).

LEMMA 5.3.

(i) Let $A$ be a semi-closure operation and $B$ be a closure operation. Then

(i $) A B=A B A$ implies $B A \leqq A B$,

(ib) $B A \leqq A B$ if and only if $A B=B A B$.

(ii) Let $A$ and $B$ be closure operations. Then $B A \leqq A B$ if and only if $A B=A B A=B A B$.

(iii) Let $A$ and $B$ be semi-closure operations. Then $A B=A B A$ and $B A=B A B$ imply $A B=B A$.

Proof. ( $\left.\mathrm{i}_{a}\right) B A \leqq A B A=A B$.

(ib) Assume $B A \leqq A B$. Since $B$ is idempotent,

$$
A B \leqq B A B \leqq(A B) B=A B^{2}=A B
$$

and hence

$$
A B=B A B .
$$

The converse is obvious. 
(ii) By $\left(\mathrm{i}_{b}\right), B A \leqq A B$ implies $A B=B A B$. Since $A$ is idempotent, $B A \leqq A B$ implies $A B A \leqq A B$; at once we have $A B=A B A$. The converse is clear.

(iii) $B A \leqq A B A=A B, A B \leqq B A B=B A$.

THEOREM 5.3. Let $\left\{P_{i} ; i=1, \cdots, n\right\}$ be a set of a finite number of join-conservative closure operations. If $P_{i} P_{j}=P_{i} P_{j} P_{i}$ for all $i, j, i \neq j$, then

$$
\left\{P_{i} ; i=1, \cdots, n\right\}^{\#}=P_{1} \cdots P_{n}=P_{i_{1}} \cdots P_{i_{n}}, i_{j} \neq i_{l}, j \neq l
$$

where $\left(i_{1}, \cdots, i_{n}\right)$ is any permutation of $1, \cdots, n$.

Proof. By Theorem 5.2,

$$
\left\{P_{i} ; i=1, \cdots, n\right\}^{\#}=\bigcup_{m=1}^{\infty}\left(P_{i_{1}} \cdots P_{i_{n}}\right)^{m} .
$$

On the other hand, by the assumption and by Lemma 5.3

$$
P_{i} P_{j}=P_{j} P_{i} \text {, for all } i, j, i \neq j \text {, and } P_{i}^{2}=P_{i} \text { for all } i
$$

and

$$
\left(P_{i_{1}} \cdots P_{i_{n}}\right)^{m}=P_{i_{1}} \cdots P_{i_{.,}} \text {for all } m \text {. }
$$

Thus we have proved (5.5).

6. Applications. First we introduce an important concept of generalized implications from which semi-closure operations are derived. A function $f$ on a nonempty set $E$ is a mapping which associates with each ordered system $\left(x_{1}, \cdots, x_{n}\right)$ of elements of $E$ a single element $f\left(x_{1}, \cdots, x_{n}\right)$ of $E$. For convenience, $f$ is considered as a function defined on the product set $E^{n}=E \times \cdots \times E$, that is

$$
f(\mathscr{X}) \in E, \quad \mathscr{X}=\left(x_{1}, \cdots, x_{n}\right) \in E^{n} .
$$

Consider another mapping $\Pi$ which associates with each $\rho$ of $\mathscr{B}$ a subset $\rho \Pi$ (possibly empty) of $E^{n}: \rho \stackrel{\Pi}{\rightarrow} \rho \Pi$ such that $\Pi$ is isotonic and join-conservative, i.e.,

$$
\rho \subseteq \sigma \text { implies } \rho \Pi \subseteq \sigma \Pi
$$

$$
\text { If }\left\{\rho_{\alpha}\right\} \text { is directed, }\left(\bigcup_{\alpha} \rho_{\alpha}\right) \subseteq \bigcup_{\alpha}\left(\rho_{\alpha} \Pi\right)
$$

where $\bigcup$ of the right side denotes the usual union. $\Pi$ is called a range-mapping of $\mathscr{B}$ into $E^{n}$.

If functions $h$ and $k$ on $E^{n}$ and a range-mapping $\Pi$ of $\mathscr{B}$ into $E^{n}$ are given, a condition on relations is associated with them as follows:

$$
\mathscr{X} \in \Pi \rho \text { implies } h(\mathscr{X}) \rho k(\mathscr{X}) \text {. }
$$

A condition of this kind is called a "generalized implication," and it is denoted 
by $((\Pi ; h, k))$. From a generalized implication $((\Pi ; h, k))$, a semi-closure operation $P$ is derived as follows:

$$
\rho P=\rho \cup \tau_{\rho}
$$

where $\tau_{\rho}=\{(h(\mathscr{X}), k(\mathscr{X})) ; \mathscr{X} \in \rho \Pi\}$, and $\tau_{\rho}=\square$ if $\rho \Pi=\square$. By (6.1) and (6.2), we can see that $P$ is a join-conservative semi-closure operation. $P$ is called the semi-closure operation derived from $((\Pi ; h, k))$. Clearly $\rho P=\rho$ iff $\rho$ satisfies the condition $((\Pi ; h, k))$. More generally, suppose a system of generalized implications

$$
\left\{\left(\left(\Pi_{\alpha} ; h_{\alpha}, k_{\alpha}\right)\right) ; \alpha \in \Delta\right\}
$$

are given and let $P_{\alpha}$ be the semi-closure operation derived from $\left(\left(\Pi_{\alpha} ; h_{\alpha}, k_{\alpha}\right)\right)$ where $\Pi_{\alpha}$ is a range-mapping of $\mathscr{B}$ into $E^{n_{\sim}}, 1 \leqq n_{\alpha}<\infty$. Then $\rho$ satisfies $\left(\left(\Pi_{\alpha} ; h_{\alpha}, k_{\alpha}\right)\right)$ for all $\alpha \in \Delta$ iff $\rho$ is $\left(\bigcup_{\alpha \in \Delta} P_{\alpha}\right)$-closed, that is $\rho$ is of type $\bigwedge_{\alpha \in \Delta} P_{\alpha}$. The important examples of generalized implications are given as follows:

Suppose we are given two systems of functions, $\left\{f_{\xi} ; \xi \in \Xi\right\}$ and $\left\{g_{\xi} ; \xi \in \Xi\right\}$ where the index sets are the same. For these sets, a range-mapping, denoted by $\Pi\left(f_{\xi}, g_{\xi}\right)$, is defined by

$$
\rho \Pi\left(f_{\xi}, g_{\xi}\right)=\left\{\mathscr{X} \in E^{n} ; f_{\xi}(\mathscr{X}) \rho g_{\xi}(\mathscr{X}) \text { for all } \xi \in \Xi\right\} \text {. }
$$

Obviously, $\Pi\left(f_{\xi}, g_{\xi}\right)$ satisfies (6.1) and (6.2). A condition of this form $\left(\left(\Pi\left(f_{\xi}, g_{\xi}\right) ; h, k\right)\right)$ is called an implication condition or implication which is also denoted by

$$
f_{\xi} \rho g_{\xi}(\text { for all } \xi \in \Xi) \Rightarrow h \rho k \text {. }
$$

As the second example, $\Pi_{0}$ is defined as follows:

$$
\rho \Pi_{0}=E^{n} \text { for all } \rho \in \mathscr{B} .
$$

The generalized implication with the range-mapping (6.6) is called identity condition or identity, which means

$$
h(\mathscr{X}) \rho k(\mathscr{X}) \text { for all } \mathscr{X} \in E^{n} \text {. }
$$

In particular if every element of $\mathscr{B}$ is reflexive, then identities are regarded as the special case of implications; and the case where $h, k, f_{\xi}$ and $g_{\xi}$ are given by words is familiar to us if $E$ is a groupoid.

EXAMPLES.

(6.8) Equivalence: $x \rho x ; x \rho y \Rightarrow y \rho x ; x \rho y, y \rho z \Rightarrow x \rho z$.

(6.9) Compatible: $x \rho y \Rightarrow z x \rho z y ; u \rho v \Rightarrow u w \rho v w$.

(6.10) Associative: $(x y) z \rho x(y z)$.

(6.11) Congruence: (6.8), (6.9). 
(6.12) Let $G$ be a groupoid and $\rho$ be a congruence such that $G / \rho$ is cancellative: $x z \rho y z \Rightarrow x \rho y, w u \rho w v \Rightarrow u \rho v$ besides (6.11).

(6.13) The factor groupoid $G / \rho$ is a semilattice: $x \rho x^{2}, y z \rho z y$ besides (6.10), (6.11).

(6.14) $G / \rho$ is weakly reductive [3]: The system of $x a_{\xi} \rho y a_{\xi}$ and $a_{\xi} x \rho a_{\xi} y$ for all $a_{\xi} \in G$ imply $x \rho y$.

(6.15) $G / \rho$ has at most one idempotent element: $x \rho x^{2}$ and $y \rho y^{2}$ imply $x \rho y$.

Let $S$ be a set in which binary operations $\theta_{\alpha}, \alpha \in \Lambda$, are defined. Then $S$ is called an algebraic system. We know many examples of implications or identities in algebraic systems: for example, distributivity with respect to rings, modularity with respect to lattices, etc.

We give a few examples of the applications of $\S 5$. First we define several operations on $\mathscr{B}_{0}$.

(6.16.1) Reflexive operation $R: \rho R=\rho \cup \mathfrak{l}$ (1 equality relation).

(6.16.2) Symmetric operation $S: \rho S=\rho \cup \rho(-1), \rho(-1)=\{(x, y) ;(y, x) \in \rho\}$.

(6.16.3) $\rho T_{2}=\rho \cup \rho^{2}, \rho^{2}=\{(a, b) ;(a, x) \in \rho,(x, b) \in \rho$ for some $x \in E\}$.

(6.16.4) Transitive operation $T: T=T_{2}^{\#}=\bigcup_{i=1}^{\infty} T_{2}^{i}$.

If $E$ is a groupoid,

$$
\begin{aligned}
& \rho C_{r}=\rho \cup\{(a x, b x) ; x \in G,(a, b) \in \rho\}, \\
& \rho C_{l}=\rho \cup\{(x a, x b) ; x \in G,(a, b) \in \rho\} .
\end{aligned}
$$

$$
\text { Compatible operation } C: C=\left\{C_{r}, C_{l}\right\}^{\#}=\bigcup_{i=1}^{\infty}\left(C_{r} C_{l}\right)^{i}=\bigcup_{i=1}^{\infty}\left(C_{l} C_{r}\right)^{i} \text {. }
$$

(6.16.7) Equivalence operation $Q: Q=\{R, S, T\}^{\#}=R S T=S R T=S T R$.

(6.16.8) Congruence operation $N: N=\{R, S, C, T\}^{\#}=R S C T=R C S T$.

The operations $R$ and $S$ are already closure operations. The forms of $T$ and $C$ are obtained by Theorem 5.2; $Q$ and $N$ are done by Theorems 5.2 and 5.3 by being aware of the fact that $\left({ }^{2}\right)$

$$
\begin{aligned}
& R S=S R, R T=T R, S T S=T S T=S T, \\
& R C=C R, S C=C S, C T C=T C T=C T .
\end{aligned}
$$

If $E$ is a semigroup, $C=C_{r} C_{l}=C_{l} C_{r}, C_{r}^{2}=C_{r}, C_{l}^{2}=C_{l}$.

(2) The structure of the semigroup generated by $R, S, T$ and one generated by $R, S, T, C$ was reported briefly in [13] but the detailed argument will be published elsewhere. 
Theorem 5.2 can be stated in most applicable form:

COROllary 6.1. Let $P$ be a semi-closure operation derived from a basic type. The relation $\sigma$ of "type $P$ " generated by $\rho$ is given by

$$
\sigma=\rho W, \quad W=\bigcup_{i=1}^{\infty}(P N)^{i}, N=R S C T .
$$

Especially if $P$ is derived from a system of identities,

$$
W=P N \text {. }
$$

Proof. The former half of the corollary is an immediate consequence of Theorem 5.2. The latter half is obtained as the special case of the former since identities are preserved by any homomorphism, that is, $P N P=P N$ and hence $(P N)^{i}=P N$ for all $i$. q. e. d.

For example, to find the smallest commutative-congruence on a given groupoid $E$, we may apply the operations to $\mathrm{l}$, the equality relation, that is, the congruence generated by the relation.

$$
\{(x y, y x) ; x, y \in E\}
$$

gives the desired one.

To find a maximal homomorphic image of type given by system of a finite number of identities, we can find a maximal homomorphic image of the type given by each identity successively in any order (Theorem 5.3).

COROLlary 6.2. Let $S$ be an algebraic system and suppose that a system of implications

$$
f_{\xi_{\eta}}=g_{\xi_{\eta}}, \eta \in \Delta, \Rightarrow h_{\xi}=k_{\xi}, \xi \in \Xi
$$

is given. Then the set of congruences $\rho$ such that $S / \rho$ satisfies (6.19) has a smallest $\left(^{3}\right)$ element.

Proof. Let $P_{\xi}$ be the semiclosure operation derived from the condition (6.19) and let $P=\bigcup_{\xi} P_{\xi}$; let $\mathrm{\imath}$ be the equality relation on $S$. By Corollary 6.1 , the smallest congruence on $S$ is given by

$$
\mathfrak{l}(P N)^{\#}=\imath \bigcup_{i=1}^{\infty}(P N)^{i} .
$$

COROLlaRY 6.3. If $S$ is an algebraic system, then the set of equivalences on $S$ all of whose equivalence classes are subalgebraic-systems of $S$ has a smallest element.

Proof. A relation $\rho$ is an equivalence relation such that each equivalence class is a subalgebraic-system if and only if (6.8) and

(3) See footnote 1. 


$$
x \rho y \Rightarrow x \rho x y .
$$

Let $P$ be the semi-closure operation derived from (6.20). Then $1(P Q)^{\#}, Q$ given in (6.16.7), is the desired one.

COROLlARY 6.4. There is a smallest compatible quasi-ordering containing a given relation.

We shall show more examples of basic types and join-types.

In (6.5), $h, k, f_{\xi}$ and $g_{\xi}$ may be given in a mixed form using both mappings and words. For example:

(6.21) Let $\phi$ be a fixed mapping of a groupoid $G$ into itself.

$$
\phi(x) x y \rho y, \quad(y x) \phi(x) \rho y .
$$

Let $P_{\xi}$ be a fixed semi-closure operation on $\mathscr{B}_{0}$. Instead of (6.4), consider

$$
\rho \Pi_{1}\left(f_{\xi}, g_{\xi}\right)=\left\{\mathscr{X} \in E^{n} ; f_{\xi}(\mathscr{X})\left(\rho P_{\xi}\right) g_{\xi}(\mathscr{X}) \text { for all } \xi \in \Xi\right\} \text {. }
$$

As a slight generalization of implications, we have the condition

$$
f_{\xi}\left(\rho P_{\xi}\right) g_{\xi}(\text { for all } \xi \in \Xi) \Rightarrow h \rho k \text {. }
$$

For example:

(6.22) Let $\rho$ be a congruence on a semigroup, $\rho P$ be the congruence of type $\{x y=y x\}$ generated by $\rho$. Consider

$$
x \rho P x y \rho P y \Rightarrow x y \rho y x .
$$

If this is applied to groups $G, \rho$ is the congruence on $G$ such that the commutator subgroup of $G$ is abelian. Similarly we have an example:

(6.23) A congruence $\rho$ on a group $G$ such that the $k$ th commutator subgroup of $G / \rho$ is abelian.

Of course Theorem 2.2 can be applied to each of the above examples. Further we add more:

Let $\rho$ be a congruence on a groupoid $G$. The following two conditions respecting $\rho$ give basic types.

(6.24) $G / \rho$ is a group such that the order of every nonidentity element of $G / \rho$ is a divisor of a fixed $n$.

(6.25) The congruence class containing a fixed element $a$ of $G$ is a right identity in $G / \rho: x a \rho x, a$ being a constant element.

On the other hand none of the following conditions is a basic type, but they are join-types. 
(6.26) $G / \rho$ has a right identity.

(6.27) $G / \rho$ is a group.

(6.28) Under the restriction of $G$ to groups, $G / \rho$ is a solvable group. This is a type joining the forms (6.22).

Finally, the author expresses his thanks to the editor and the referee for their kind advice on the publication of this paper, and also to Professor Clifford for his personal letter informing me of his useful suggestions.

Added Note. After writing this paper, I read Hammer's unpublished paper, Extended topology: structure of isotonic functions, in which he studies isotonic functions from the standpoint of topology, and his terminology "enlarging" and "expansive" are the same as my "extensive" and "semi-closure," respectively.

\section{REFERENCES}

1. G. Birkhoff, Lattice theory, rev. ed., Amer. Math. Soc. Colloq. Publ. Vol. 25, Amer. Math. Soc., Providence, R. I., 1948.

2. R. H. Bruck, A survey of binary systems, Springer, Berlin, 1958.

3. A. H. Clifford and G. B. Preston, Algebraic theory of semigroups, Math. Survey No. 7, Amer. Math. Soc., Providence, R. I., 1961.

4. N. Kimura, On some existence theorems on multiplicative system 1, greatest quotient, Proc. Japan Acad. 34 (1958), 305-309.

5. O. Ore, Some studies on closure relations, Duke Math. J. 10 (1943), 761-785.

6. M. Petrich, The maximal semilattice decomposition of a semigroup, Bull. Amer. Math. Soc. 66 (1963), 342-344.

7. G. B. Preston, Congruences on completely 0-simple semigroups, Proc. London Math. Soc. 11 (1961), 557-576.

8. T. Tamura and N. Kimura, On decompositions of a commutative semigroup, Ködai Math. Sem. Rep. 4 (1954), 182-225.

9. —- Existence of greatest decomposition of a semigroup, Kōdai Math. Sem. Rep 7 (1955), 83-84.

10. T. Tamura, Notes on finite semigroups and determination of semigroups of order $4, \mathbf{J}$. Gakugei Tokushima Univ. 5 (1954), 17-27.

11. —- All semigroups of order at most 5, J. Gakugei Tokushima Univ. 6 (1955), 19-39.

12. - The theory of construction of finite semigroups 1, Osaka Math. J. 8 (1956), 243-261.

13. - Operations on binary relations and their applications, Bull. Amer. Math. Soc. 70 (1964), 113-120.

14. M. Yamada, On the greatest semilattice decomposition of a semigroup, Kōdai Math. Sem. Rep. 7 (1955), 59-62.

15. A. H. Clifford, Semigroups admitting relative inverses, Ann. of Math. 42 (1941), 1037-1049.

16. D. McLean, Idempotent semigroups, Amer. Math. Monthly 61 (1954), 110-113.

Universtry of California, Davis, California 